\title{
Éthique publique
}

Revue internationale d'éthique sociétale et gouvernementale

vol. $17, \mathrm{n}^{\circ} 1 \mid 2015$ :

Penser l'ouverture des frontières

\section{Pourquoi penser l'ouverture des frontières}

\author{
IDIL ATAK ET SPERANTA DUMitRU
}

\section{Texte intégral}

$\mathrm{Au}$ XIXe siècle, il était plus facile de traverser l'Atlantique qu'il ne l'est aujourd'hui de traverser la Méditerranée. Si la traversée prenait davantage de temps, le prix du voyage et le nombre de migrants n'avaient rien de comparable avec l'actuelle traversée de la Méditerranée. En 1903 par exemple, plus de 12 ooo migrants pouvaient arriver en une seule journée dans le seul port d'Ellis Island ${ }^{1}$. Les migrants européens s'entassaient par milliers dans l'entrepont des bateaux payant l'équivalent de 175 à 275 dollars pour une traversée pénible de 8 à 14 jours. On estime à plus de 55 millions le nombre de migrants européens qui ont ainsi traversé l'Atlantique entre 1840 et 1914 (Hatton et Williamson, 1998 ; Ferenczi et Willcox, 1929). À ceux-ci, il faut ajouter quelque 100 millions de migrants vers le sud et le nord de l'Asie (McKeown, 2004). Un siècle plus tard, avec 100 millions de migrants de plus pour une population mondiale trois fois plus élevée, les migrations sont vues comme un problème nécessitant de nouvelles régulations en dépit de la fermeture drastique des frontières.

Les chercheurs considèrent l'année 1914 comme la fin de l'âge de la migration de masse $^{2}$. Le début de la Grande Guerre a eu pour effet l'introduction du régime de passeports obligatoires, qui n'existait plus que dans certains pays (l'Empire ottoman, la Russie, la Roumanie, la Bulgarie, etc.). En 1914, les pays belligérants, la France, l'Allemagne, l'Italie furent les premiers à introduire l'obligation des passeports, suivis en 1916-1917 par l'Espagne, la Suisse, le Danemark. En 1919, le traité de Versailles annonce la création de la Société des Nations et mentionne, dans son article 23(e), le rétablissement de la liberté « de transit » de l'avant-guerre. Affirmant que la paix durable ne peut être assurée sans justice sociale, le Pacte prévoit la création d'une organisation permanente, chargée de défendre les droits des travailleurs, y compris des « travailleurs occupés à l'étranger ». Pour cela, le Bureau 
international du Travail constituera une Commission de l'émigration internationale, tandis que les participants de la Conférence de Paris en 1920 discuteront de la mise en place d'un passeport uniforme, identique pour tous les pays, qui faciliterait la liberté de transit pour les travailleurs, tout en permettant un contrôle plus rapide par les autorités.

3 Un siècle après, non seulement les vœux des signataires du traité de Versailles semblent oubliés à jamais, mais les signataires de la Déclaration universelle des droits de l'homme', qui s'engageaient en 1948 à garantir le droit de « toute personne [...] de quitter tout pays, y compris le sien ${ }^{4}$ », signent aujourd'hui des accords limitant l'exercice de ce droit. L’idée de faciliter la liberté de transit par un passeport uniforme est devenue incongrue dans un monde où même le droit à chercher asile lorsque son pays est en guerre fait l'objet de mépris. Aujourd'hui, ces engagements sont si peu respectés que les autorités européennes envisagent, publiquement et sans aucune réserve, de détruire les bateaux de ceux qui s'apprêtent à exercer leurs droits $^{5}$. Nombre de personnes qui essaient de contourner les restrictions considérables à la liberté de circulation et au droit de chercher asile risquent leur vie ou décèdent avant d'arriver à destination. Celles qui arrivent à bon port se retrouvent souvent en situation de vulnérabilité et aucun pays " développé » n’a signé la Convention internationale sur la protection des droits de tous les travailleurs migrants et des membres de leur famille.

4 Ce dossier d'Éthique publique propose une réflexion sur un siècle de contrôles accrus des frontières par un effort d'imagination morale : quelles seraient les conséquences politiques, juridiques, sociales, culturelles, économiques de la réouverture des frontières, et comment les analyser d'un point de vue éthique ? Cette réflexion est déjà engagée dans les milieux de la recherche et de la politique internationale. Ainsi, les économistes ont-ils calculé qu'une augmentation de $3 \%$ de la migration de travail vers les pays riches ferait gagner aux pays pauvres deux fois plus qu'ils n'obtiendraient par plusieurs politiques de développement conjuguées (Pritchett, 2006), tandis qu'une ouverture totale des frontières augmenterait considérablement le produit intérieur brut mondial (Clemens, 2011) et doublerait celui des pays en développement (Kennan, 2013). De même, les Nations Unies ont tenté d'introduire la migration au Programme de développement pour l'après-2015 (Dumitru, 2013). De nombreuses organisations non gouvernementales soulignent l'économie aussi bien budgétaire et en nombre de vies humaines qu'apporterait une ouverture des frontières.

5 Toute réflexion sur l'ouverture des frontières se heurte nécessairement à des concepts de souveraineté nationale et de frontières, y compris lorsque l'on envisage la gouvernance mondiale des migrations. La liberté de circulation, la justice, le cosmopolitisme, l'hospitalité et la citoyenneté constituent autant de fils conducteurs pour réfléchir aux nouveaux défis éthiques. Plusieurs articles de ce numéro de la revue Éthique publique explorent le sens et l'évolution de ces concepts dans un contexte de mondialisation et de contrôle des migrations.

$6 \quad$ Magali Bessone observe que les difficultés à penser l'ouverture des frontières sont profondes. Dans son article, elle pose un regard critique sur les discours et les politiques qui conceptualisent l'immigration en termes d'hospitalité et d'accueil, créant ainsi une dichotomie entre citoyens et étrangers et ayant pour effet d'exclure les étrangers de la sphère légitime du politique et des droits. L'auteure montre comment la conceptualisation de l'immigration en tant qu'hospitalité, c'est-à-dire une norme de bienveillance qui régit la sphère privée, permet de justifier le maintien, voire le durcissement, de rapports différenciés à un territoire préalablement défini comme lieu d'appartenance des accueillants. Ce plaidoyer pour la mise en œuvre effective de la non-discrimination fondée sur le statut légal, engage aussi une réflexion sur le concept de citoyenneté. Bessone souligne qu'il est important de donner à toute personne résidant sur le territoire d'un État et désireuse d'être 
engagée dans la conversation démocratique sur les normes de la citoyenneté, la capacité légale et politique de le faire.

$7 \quad$ L'article d'Alessandra Sciurba et Filippo Furri fait écho à celui de Bessonne en ce qu'il critique le biais exclusivement humanitaire dans la compréhension des droits à la libre circulation, assorti d'une vision de l'immigration comme une situation rare et exceptionnelle. Les auteurs décrivent les conditions intellectuelles qui ont conduit à la rédaction de la Charte de Lampedusa affirmant une liberté de circulation égale pour toutes et tous. Bien que motivée par le naufrage de 368 personnes souhaitant traverser la Méditerranée en octobre 2013, la Charte de Lampedusa a été inspirée par une pensée radicalement égalitaire en matière de droits à la mobilité. Les auteurs plaident pour une façon alternative de penser les droits de l'homme "par le bas » et qui ne dépende pas de leur reconnaissance par les États. Cette vision se trouve en partie accomplie dans l'initiative associative transnationale qui a présidé à la rédaction de la Charte.

8 L'analyse d'Isabelle Sauriol consolide cette vision. Dans son article, elle montre que le droit à la mobilité dans le cadre d'une ouverture des frontières à l'échelle internationale ne serait pas incompatible avec un droit à la résidence permanente. Sauriol met l'accent sur le fait que toute personne est porteuse de droits et doit pouvoir les exercer là où elle réside, travaille et vit. L'exercice effectif de droits est, comme le note l'auteure, le fondement même de la démocratie. L'article propose un examen comparatif du cadre juridique gouvernant la résidence au Canada et aux États-Unis, ainsi que dans le contexte de l'Union européenne et des nouveaux pays d'accueil du Sud. Il met au jour les problèmes et les défis liés à l'accès des migrants aux droits attachés à la résidence. Sauriol examine également des solutions pouvant améliorer le statut des résidents non citoyens. Elle préconise, entre autres, une forme de « citoyenneté de résidence » basée sur la reconnaissance de droits fondamentaux de migrants, y compris un droit effectif à l'égalité, les droits politiques tels que le droit de vote, et le traitement national en matière de droits économiques et sociaux.

De son côté, Louis Lourme explore le potentiel du cosmopolitisme pour défendre une ouverture des frontières. Il analyse plus précisément le type d'« ouverture » des frontières suggéré par le point de vue cosmopolitique sur les plans moral et politique. L'auteur remarque que la reconnaissance des frontières nationales sur les plans historique et politique est constitutive du cosmopolitisme, et que ce dernier peut articuler la réalité des frontières avec les innovations institutionnelles permettant de les ouvrir. Selon Lourme, le cosmopolitisme reflète l'interdépendance humaine et la porosité des frontières dans un monde où la justice à l'intérieur des frontières est inséparable de la justice au-delà des frontières. En ce sens, le cosmopolitisme correspond, sur le plan moral traditionnel comme sur le plan du projet politique, à une conception de la frontière ouverte.

Antoine Pécoud, pour sa part, propose une analyse critique de la " gouvernance mondiale des migrations » et notamment de la place de la liberté de circulation dans les politiques migratoires. Il explique comment les politiques migratoires font l'objet d'une concertation et de coopération accrues entre États, et que des recommandations élaborées dans des enceintes intergouvernementales telles que l'ONU participent à l'émergence d'une gouvernance mondiale. Ces politiques et recommandations adoptent généralement une approche pro-immigration pour des raisons démographiques et économiques, et reconnaissent l'importance de refonder les politiques migratoires sur la base de principes universels comme le développement ou les droits de l'homme. Toutefois, comme le note Pécoud, peu de place est accordée à la liberté de circulation dans les débats relatifs à la gouvernance mondiale des migrations. La possibilité du choix de migrer comme une décision autonome relevant de la liberté de circuler n'y est pas prise en compte. La mobilité reste un objet de contrôle par les États. Si les migrations internationales constituent par définition une réalité transnationale, elles sont toujours considérées comme un 
problème national, qui relève de la souveraineté de chaque État. Partant de ces constats, Pécoud examine les défis liés à la mise en œuvre d'un régime de gouvernance des migrations et comment ce régime s'articule avec les mesures de contrôle des flux migratoires.

La centralité des droits de l'homme, et notamment de la liberté de circulation, dans les débats éthiques sur l'ouverture des frontières est affirmée avec force par Jean Rousseau, président de l'Organisation pour une citoyenneté universelle (O.C.U.). Rousseau présente les valeurs défendues par son organisation fondée en février 2013 et les activités menées en matière de mobilité des personnes. Il remarque l'absence de vision actuelle qui se double de la négation du droit fondamental à la libre circulation. Alors que les pays les plus privilégiés offrent à leurs citoyens des possibilités de circulation quasiment sans limites, les trois quarts de l'humanité ne peuvent échapper à une forme d'assignation à résidence de fait. Rousseau dénonce la fermeture et la militarisation des frontières, notamment européennes, nordaméricaines et australiennes qui se révèlent être des dispositifs meurtriers pour des milliers de personnes depuis plus de deux décennies. L'auteur propose repenser les politiques migratoires pour éviter que la liberté de circulation elle-même ne soit source de nouvelles formes de précarisation ou d'exploitation. À cette fin, il suggère l'adoption d'une perspective de citoyenneté universelle effective basée sur l'égal accès des migrants aux droits et libertés fondamentaux et le respect de ces droits et libertés.

Micheline Labelle se réfère aux concepts de frontières et du transnationalisme pour analyser le courant de la mobilité. Elle estime que ce courant ne rend pas compte de la complexité et de la diversité du phénomène migratoire ni des modes d'incorporation dans les pays de départ et dans les pays d'accueil. Son article propose une réflexion sur la mobilité qui prendrait en considération plusieurs facteurs, y compris les déséquilibres historico-structurels, d'ordre économique, politique et culturel, qui incitent à l'émigration dans les pays de départ, ainsi que les structures économiques et politiques qui connectent les aires et les systèmes régionaux de migration, et les liens coloniaux et postcoloniaux qui s'inscrivent dans la durée. Une telle réflexion nécessite, selon l'auteure, non seulement la caractérisation des forces structurelles d'appel dans les pays de destination, mais aussi la prise en compte de facteurs tels que les réseaux migratoires, familiaux et communautaires, les motivations individuelles, ainsi que la perspective du droit et de l'éthique.

13 Ce numéro d'Éthique publique inclut des articles qui analysent la question de l'ouverture des frontières dans une perspective régionale. Leurs auteurs expliquent comment les régions et les territoires transfrontaliers peuvent constituer des laboratoires pour l'ouverture des frontières et l'intégration sociospatiale des populations, tout en explorant les défis éthiques liés à ce processus. Christian-Yann Messe Mbega examine le cas du Sud-Cameroun, du Nord-Gabon et de la partie continentale de la Guinée équatoriale pour montrer comment l'effacement des frontières hermétiques dans les régions frontalières conduit à l'interaction spatiale, socioculturelle, économique qui réunit dans une même communauté des espaces nationaux auparavant bornés. Cette communauté d'espace ou région transfrontalière permettrait l'intégration sociospatiale de la population vivant de part et d'autre des frontières nationales, grâce aux valeurs éthiques qu'elle partage.

Caroline Caplan, de son côté, se penche sur le cas de Paso de los LibresUruguayana, ensemble urbain transfrontalier entre l'Argentine et le Brésil, qui de plus fait partie du MERCOSUR, en vue de comprendre l'intérêt qu'ont ces États et le MERCOSUR d'ériger cette frontière en territoire de vie et d'en définir les limites. Son analyse montre que les territoires restent soumis aux doctrines nationalistes des États et que la construction de territoires transfrontaliers s'en trouve limitée. Ainsi Caplan constate-t-elle que les politiques protectionnistes des États, veillant à faire appliquer les lois nationales à Paso de los Libres, complexifient le passage de la 
frontière et diminuent les échanges entre villes jumelles, notamment dans le secteur de l'éducation. La frontière entre l'Argentine et le Brésil resterait avant tout une frontière pensée pour la circulation des marchandises. L'auteure remarque que le défaut d'intégration sociale freine potentiellement la croissance économique régionale.

Ces exemples mettent en lumière les défis politiques, économiques et sociaux que soulève l'ouverture des frontières. Comme le note Caplan, effacer les frontières requiert l'existence de systèmes de gouvernance adaptés, qui dépassent le cadre national. Les territoires transfrontaliers mettent donc au défi les États-nations et les obligent à repenser le territoire et la souveraineté. Les deux articles en question illustrent également comment des régions transfrontalières peuvent devenir des lieux d'apprentissage sur le plan de la gouvernance et de la coopération décentralisée.

Pour terminer, Mathilde Unger se penche sur le cas de l'Union européenne qui offre le modèle régional le plus poussé au monde du point de vue de la liberté de circulation, pour souligner les défis sociaux que représente l'ouverture des frontières. Elle explique d'une part, comment la jurisprudence de la Cour de justice de l'Union européenne relative au droit de circuler et de résider librement à l'intérieur de l'Union a précisé la dimension sociale de la citoyenneté européenne. Ainsi, comme le souligne l'auteure, le droit communautaire reconnaît au citoyen européen séjournant dans un autre État membre un statut qui lui permet d'accéder à plusieurs droits socioéconomiques au même titre que les nationaux de cet État membre. Toutefois, Unger montre d'autre part que ces droits sont souvent soumis à des dérogations qui en limitent la portée, et que l'ouverture des frontières place inévitablement les États dans une situation de concurrence normative qui ne profite pas aux modèles sociaux les plus généreux.

17 En somme, les auteurs de ce numéro spécial montrent, directement ou indirectement, combien il est difficile aujourd'hui de penser l'ouverture des frontières dans un monde construit sur le postulat opposé. On pourrait dire qu'il suffit d'un siècle non seulement pour enraciner des pratiques politiques contraires aux libertés individuelles fondamentales, mais aussi pour perdre collectivement la capacité à imaginer des voies de sortie.

\section{Bibliographie}

CHARIE DE LAMPEDUSA (2014). En ligne à http://www.lacartadilampedusa.org/pdf_carta /charte\%2ofranccais.pdf, consulté le 31 mai 2015.

Clemens, Michael (2011), " Economics and emigration : Trillion-dollar bills on the sidewalk? », The Journal of Economic Perspectives, vol. 25, no 3, p. 83-106.

DOI : 10.1257/jep.25.3.83

Dumitru, Speranta (2013) « Des visas, pas de l'aide ! De la migration comme substitut à l'aide au développement », Éthique publique, vol. 15, no 2, p. 77-98.

DOI : $10.4000 /$ ethiquepublique.1231

FERENCZI, Imre, et Walter Willcox (1929), International Migration, Volume I : Statistics,New York, NBER.

Hatton, Timothy, et Jeffrey G. Williamson (1998), The Age of Mass Migration, New York, Oxford University Press.

KenNAN, John (2013), « Open borders », Review of Economic Dynamics, vol. 16, no 2, p. L1L13.

DOI : 10.1016/j.red.2012.08.003

OFFICE QUÉBÉCOIS DE LA LANGUE FRANÇAISE (2005). Le grand dictionnaire terminologique, En ligne à gdt.oqlf.gouv.qc.ca, consulté le 8 juin 2015 .

LE MONDE (2015), « Migrants : l'UE met sur pied une opération navale contre les trafiquants en Méditerranée » 18 mai.

McKeown, Adam (2004), " Global Migration 1840-1945 », J ournal of World History, vol. 15, no 2, p. 155-189. 
Nations Unies (1948), Déclaration universelle des droits de l'homme. En ligne à http://www.un.org/fr/documents/udhr/, consulté le 8 juin 2015.

PRITchetT, Lant (2006), Let Their People Come : Breaking the Gridlock on Global Labor Mobility, Washington DC, Center for Global Development.

THE NEWYORK TIMES(1903), « Big Army of Immigrants ; Nine ships bring 12,668 Foreigners from European Ports », 3 avril.

\section{Notes}

1 Voir par exemple l'article dans The New York Times du 3 avril 1903, " Big Army of Immigrants: Nine ships bring 12,668 Foreigners from European Ports ».

2 Voir Adam McKeown (2004) pour une remise en cause de cette datation euro-centrée.

3 De nombreux auteurs utilisent également «droits humains ». Néanmoins, le terme « droits de l'homme », retenu par l'ensemble des institutions des pays francophones, fut d'abord choisi par les Nations Unies (ex. : Déclaration universelle des droits de l'homme, 10 déc. 1948 ; HautCommissariat aux droits de l'homme ; Commission des droits de l'homme). La Commission française consultative des droits de l'homme a adopté le 19 décembre 1998 un avis indiquant clairement la spécificité et la pertinence du terme "droits de l'homme » sur les plans historique et linguistique (OQLF, 2005).

4 Article 13(2) de la Déclaration universelle des droits de l'homme.

5 Voir par exemple l'article paru le 18 mai 2015 dans le journal Le Monde, « Migrants : l'UE met sur pied une opération navale contre les trafiquants en Méditerranée ».

\section{Pour citer cet article}

Référence électronique

Idil Atak et Speranta Dumitru , « Pourquoi penser l'ouverture des frontières », Éthique publique [En ligne], vol. 17, n 1 | 2015, mis en ligne le 18 juin 2015, consulté le 14 juillet 2017. URL : http://ethiquepublique.revues.org/1727

\section{Auteurs}

\section{Idil Atak}

Idil Atak est docteure en droit et professeure adjointe au Département de criminologie de l'Université Ryerson. Présidente de l'Association canadienne d'études sur les réfugiés et la migration forcée (ACERMF), elle est également associée de recherche à la Chaire Hans et Tamar Oppenheimer en droit international public de l'Université McGill. Elle enseigne des cours d'Éthique en justice criminelle, inégalités sociales et migrations, sécurité et droits humains. Idil Atak mène actuellement une recherche subventionnée par le Conseil de recherches en sciences humaines $(\mathrm{CRSH})$, explorant la criminalisation de la migration et de l'asile au Canada et en Europe.

\section{Speranta Dumitru}

Speranta Dumitru est maîtresse de conférences en Science politique à la Faculté de droit de I'Université Paris Descartes et membre du CERLIS, CNRS. Elle dirige le projet de recherche «Migration et développement humain : opportunités et discriminations de la personne migrante en demande de régularisation " (PICRI, IdF, 2014-2017) et l'équipe Paris Descartes du projet « Théories politiques de l'immigration et nationalisme méthodologique » (USPC, 2013-2016). Dans ses travaux, elle cherche à comprendre comment la perception de certaines formes de mobilité comme " migration » change nos cadres habituels de pensée. Le dernier numéro de revue qu'elle a édité s'intitule Les sciences sociales sont-elles nationalistes ? (Raisons politiques, 2014).

\section{Articles du même auteur}

Des visas, pas de l'aide ! De la migration comme substitut à l'aide au développement [Texte intégral]

Paru dans Éthique publique, vol. 15, n 2 | 2013 


\section{Droits d'auteur}

Tous droits réservés 